\title{
A Simulated Annealing Approach to Supplier Selection Aware Inventory Planning
}

\author{
Seda Türk, Simon Miller, Ender Özcan, Robert John \\ ASAP Research Group, School of Computer Science, University of Nottingham, UK \\ \{seda.turk,s.miller,ender.ozcan,robert.john\}@ nottingham.ac.uk
}

\begin{abstract}
Selection of an appropriate supplier is a crucial and challenging task in the effective management of a supply chain. Also, appropriate inventory management is critical to the success of a supply chain operation. In recent years, there has been a growing interest in the area of selection of an appropriate vendor and creating good inventory planning using supplier selection information. In this paper, we consider both of these tasks in a two-stage approach employing Interval Type-2 Fuzzy Sets (IT2FS) and Simulated Annealing (SA). In the first stage, the supplier selection problem is solved by using IT2FS for ranking the suppliers. We present an inventory model incorporating information from the first stage that captures the influence of supplier risk on the total cost of supply chain operation. In the second stage, $\mathrm{SA}$ is used for solving the inventory planning problem based on this model improving on both supply chain operation cost and supplier risk. In this study, we evaluated our approach using different scenarios and scalarisation techniques for SA to handle two objectives, simultaneously.
\end{abstract}

\section{INTRODUCTION}

For all commercial organisations, the flow of materials and the relationships between suppliers, manufacturers, warehouses, customers and other facilities are critical to their success, and to them remaining competitive in an increasingly demanding business environment. Supply Chain Management (SCM) is the end-to-end management of the flow of materials, information and the relationships between all partners, from the procurement of raw materials to the delivery of the end product [24].

Two key aspects of SCM are i) maintaining a small number of reliable suppliers, and ii) effectively allocating resources within the supply chain through inventory planning. Achieving both of these goals reduces the chances of defective products and late delivery, while minimising costs associated with holding stock and inability to satisfy orders.

\section{A. Supplier Selection}

Evaluating and selecting suppliers is a difficult problem that is essential to ensuring that all partners within a supply chain are able to supply high quality items and minimal cost. Failure to achieve this results in an inefficient and uncompetitive operation that is unlikely to succeed. In light of this, supplier evaluation and selection are of ongoing interest within the operations management research community. Ho et al. [7] provided an overview of 78 articles published in the period 2000

978-1-4799-7492-4/15/\$31.00 (C)2015 IEEE
- 2008 focusing on the methods used, the criteria selected for evaluation and the effectiveness of the methods, ranging from mathematical programming and analytic hierarchy process to case-based reasoning and genetic algorithm (GA).

Product quality, delivery, price, manufacturing capacity and quality of service are the most commonly used evaluation criteria for selecting suppliers (Ho et al. [7]). Hence, this problem is often considered as a Multiple Attribute Decision Making (MADM) problem in which the decision involves a number of alternative suppliers who are selected based upon the decision maker's constraints and preference priorities [1]. Wu and Chen [26] tackled the decision making process under uncertainty, introduced by the Multiple Attribute Group Decision Making (MAGDM) problems, by using a linguistic weighted arithmetic averaging method that is able to deal with linguistic preference values for each decision criteria.

Previous work on MADM showed that Type-2 Fuzzy Logic is an appropriate method for dealing with the uncertainties involved in modelling the decision making process. Chen and Lee [3] have conducted a series of studies on MADM using Type-2 Fuzzy Sets (T2FS) in which they proposed an approach using ranking values and arithmetic operations of T2FS [2], presented a T2FS TOPSIS method [3] and showed how Fuzzy Multiple Attribute Group Decision Making (FMAGDM) problems can be addressed using a method that ranks Interval Type-2 Fuzzy Sets (IT2FS). Gong [5] presented an IT2FS approach to FMAGDM problems in which the weightings of the selection criteria are unknown.

Ordoobadi [19] proposed a method for computing fuzzy scores for the suppliers, taking the selection criteria into account. In this approach, suppliers are scored using criteria modelled with Type-1 Fuzzy Sets (T1FS). The fuzzy scores for each supplier are then defuzzified and used to rank suppliers in order of preference. The supplier with the highest ranking is then chosen. The work also provides more information about suppliers to the decision maker with regard to rating and selection of the appropriate suppliers.

\section{B. Inventory Management}

Inventory management is an integrated approach to plan and control inventory while considering the whole network from suppliers to end users. Efficient inventory management is critical to the success of companies. Miller et al. [16] [17] 
state that supply chains with well managed inventory avoid stock outs - where nodes within the chain are unable to satisfy demand, and holding costs - where stock is stored from one period to the next.

According to Parhizkari et al. [21], recently, there has been a growing interest in the area of selection of an appropriate vendor along with good inventory planning. In their work, a multi-objective technique is used to find the best supplier while considering inventory management.

Rezaei and Davoodi [22] proposed a multi-item inventory model considering supplier selection with respect to the quality of products. In their model, the important issue is to decide what products to order with which supplier and in which periods with determined quantities. GA is used to obtain nearoptimal solutions to the problem.

Ghodsypour and O'Brien [4] addressed a multiple sourcing problem which takes into account components of inventory management, such as, the cost of logistics, storage costs and qualitative factors of suppliers. Mohammaditabar et al. [18] extended the work of Ghodsypour and O'Brien [4] by allocating orders to selected suppliers. They also provided a joint order process in order to decrease the overall cost. The inventory items are categorised into several groups for their effective management with minimum storage and inventory cost. It has been observed that the proposed approach reduces the overall cost of logistics, including inventory holding and ordering costs, considerably.

As mentioned above, few studies have investigated supplier selection informed inventory planning. In this study, we describe an integrated two-stage fuzzy embedded approach to deal with both supplier selection and inventory planning of a supply chain problem. In order to rank suppliers, in the first stage, the supplier selection problem is dealt with using IT2FS. In the second stage, an inventory model that incorporates information from the first stage is developed to capture the influence of supplier risk on the total cost of supply chain operation. We apply a simulated annealing (SA) approach to the problem balancing the trade-off between supply chain operational cost and supplier risk. Five different scenarios are produced reflecting the attitude of "users" to the cost versus overall cost trade-off through their weighting and the performance of the proposed approach is evaluated on those scenarios.

The rest of paper is organised as follows. In section II, background on Type-2 Fuzzy Logic (T2FL) and SA are provided. Section III provides the description of the problem and the proposed solution method. In section IV, numerical experiments and results are presented. Section V, concludes the study and discusses some potential future research directions.

\section{PRELIMINARIES}

This section introduces the techniques that have been used in this study and an overview of related work from the scientific literature.

\section{A. Type-2 Fuzzy Logic (T2FL)}

Fuzzy Logic is a method of reasoning with the uncertain data, and is based upon Fuzzy Set Theory [27], which itself is an extension of traditional (crisp) set theory. Fuzzy Sets provide the means to represent data using intuitive linguistic variables rather than using 'crisp' values that do not take uncertainty into account, and therefore, may be too restrictive. For example, fuzzy sets allow us to describe qualitative statements such as 'The risk associated with Supplier A is High' or 'The delivery time for Product A is about $n$ days'. Traditional Fuzzy Logic is also referred to as Type-1 Fuzzy Logic (T1FL). A limitation of T1FL systems is that they represent uncertainty in a 'non-fuzzy' way, i.e., an element's degree of membership to a Type-1 Fuzzy Set (T1FS) is represented with a value in $[0,1]$. Mendel and John [11] pointed out that the way of T1FL leads to a number of sources of uncertainty that are not represented by T1FS, including:

1) The perception of particular words can vary. That is, words can mean different things to different people.

2) If a group of experts do not agree, the consequent of a fuzzy system may have a histogram of values associated with them.

3) The inputs to a T1FL system may be noisy, and therefore uncertain.

4) The data used to tune the parameters of a T1FL system may be noisy.

All of these problems are addressed by Type-2 Fuzzy Sets (T2FS) [27], which represent membership degrees using T1FSs providing a secondary degree of freedom to model the additional uncertainty associated with T1FSs [11]. However, the 3D fuzzy sets produced when using T2FS are extremely complex, and can be difficult to understand and use in practical applications.

Because of this complexity computationally simpler Interval Type-2 Fuzzy Logic (IT2FL) systems have been the focus of applications of Type-2 Fuzzy Logic to date [6]. Interval Type-2 Fuzzy Sets (IT2FS) restrict the secondary membership function to be either 0 or 1 . This maintains the ability to model uncertainty about membership, while significantly reducing the complexity of reasoning using T2FS [12].

In the research described here, we will use IT2FSs to represent the uncertainties inherent in the problem of supplier selection.

1) Basic Concepts of IT2FS: In this section, we review the concept of IT2FS as described by Mendel et al. [12].

Definition II.1. A Type-2 fuzzy set $\tilde{A}$ in the universe of discourse $X$ can be represented by a Type- 2 membership function $\mu_{\tilde{A}}$ shown as follows:

$$
\tilde{A}=\left((x, u), \mu_{\tilde{A}}(x, u)\right) \mid \forall x \in X, \forall u \in J_{x} \subseteq[0,1]
$$

where $x \in X$ and $u \in J_{x} \subseteq[0,1]$ in which $0 \leq \mu_{\tilde{A}}(x, u) \leq 1$. As the primary membership function is between 0 and 1 , thus 
can be expressed as:

$$
\tilde{A}=\int_{x \in X} \int_{u \in J_{x}} \mu_{\tilde{A}}(x, u) /(x, u) J_{x} \subseteq[0,1]
$$

where $\iint$ denotes a union over all admissible $x$ and $u$ [12].

Definition II.2. Let $\tilde{A}$ be a Type- 2 fuzzy set in the universe of discourse $X$ represented by the Type- 2 membership function $\mu_{\tilde{A}}$. If all $\mu_{\tilde{A}}(x, u)=1$ for $\forall x \in X$ and $u \in J_{x} \subseteq[0,1]$, then $\tilde{A}$ is called an interval type- 2 fuzzy set, shown as followings:

$$
\tilde{A}=\int_{x \in X} \int_{u \in J_{x}} 1 /(x, u) J_{x} \subseteq[0,1]
$$

where $J_{x} \subseteq[0,1]$, i.e. [12].

Definition II.3. The upper and lower membership functions an IT2FS are both Type-1 membership functions, respectively [12].

A trapezoidal IT2FS $\tilde{A}_{i}$ in the universe of discourse $X$ represented by;

$$
\begin{aligned}
\tilde{A}_{i}=\left(\tilde{A}_{i}^{U}, \tilde{A}_{i}^{L}\right)= & \left(\left(a_{i 1}^{u}, a_{i 2}^{u}, a_{i 3}^{u}, a_{i 4}^{u} ; h_{1}\left(\tilde{A}_{i}^{U}\right), h_{2}\left(\tilde{A}_{i}^{U}\right)\right),\right. \\
& \left(a_{i 1}^{l}, a_{i 2}^{l}, a_{i 3}^{l}, a_{i 4}^{l} ; h_{1}\left(\tilde{A}_{i}^{L}\right), h_{2}\left(\tilde{A}_{i}^{L}\right)\right)(4)
\end{aligned}
$$

where $\tilde{A}_{i}^{U}$ and $\tilde{A}_{i}^{L}$ are T1FS, $a_{i 1}^{u}, a_{i 2}^{u}, a_{i 3}^{u}, a_{i 4}^{u}, a_{i 1}^{l}, a_{i 2}^{l}, a_{i 3}^{l}, a_{i 4}^{l}$ are the reference points of the IT2FS $\tilde{A}_{i}, h_{j}\left(\tilde{A}_{i}^{U}\right)$ denotes the membership value of the element $a_{i(j+1)}^{u}$ in the upper trapezoidal membership function $\tilde{A}_{i}^{U}$ while $1 \leq j \leq 2$, $h_{j}\left(\tilde{A}_{i}^{L}\right)$ denotes the membership value of the element $a_{i(j+1)}^{l}$ in the lower trapezoidal membership function $\tilde{A}_{i}^{L}$ while $1 \leq$ $j \leq 2, h_{1}\left(\tilde{A}_{i}^{U}\right) \in[0,1], h_{2}\left(\tilde{A}_{i}^{U}\right) \in[0,1], h_{1}\left(\tilde{A}_{i}^{L}\right) \in[0,1]$, $h_{2}\left(\tilde{A}_{i}^{L}\right) \in[0,1], 1 \leq i \leq n$. The height of each constituent membership function is not explicitly defined as it is assumed to be equal to 1 .

\section{B. Simulated Annealing}

Many real-world problems are too complex and exact methods often fail in providing 'high quality' solutions in a 'reasonable' amount of time. Heuristics, metaheuristics and hyper-heuristics are alternative methods in problem solving. Simulated Annealing (SA) is a well-known iterative metaheuristic, inspired from the annealing process in the metallurgical industry, for solving computationally difficult optimisation problems (Metropolis et al. [13], Kirkpatrick et al. [10]). It has been successfully applied to many different real-world optimisation problems. For example, Miller et al. [15] proposed a method that combined Interval Type-2 Fuzzy Logic and SA to investigate inventory management problems in realworld systems. Burke et al. [8] proposed SA-based hyperheuristics that combine different methods for mixing multiple neighbourhood operators and simulated annealing as a move acceptance method. They tested their approaches across a variety of problem domains ranging from timetabling to bin packing.

Algorithm 1 presents the pseudo-code of the approach [13], [10], [23]. This approach starts with a randomly

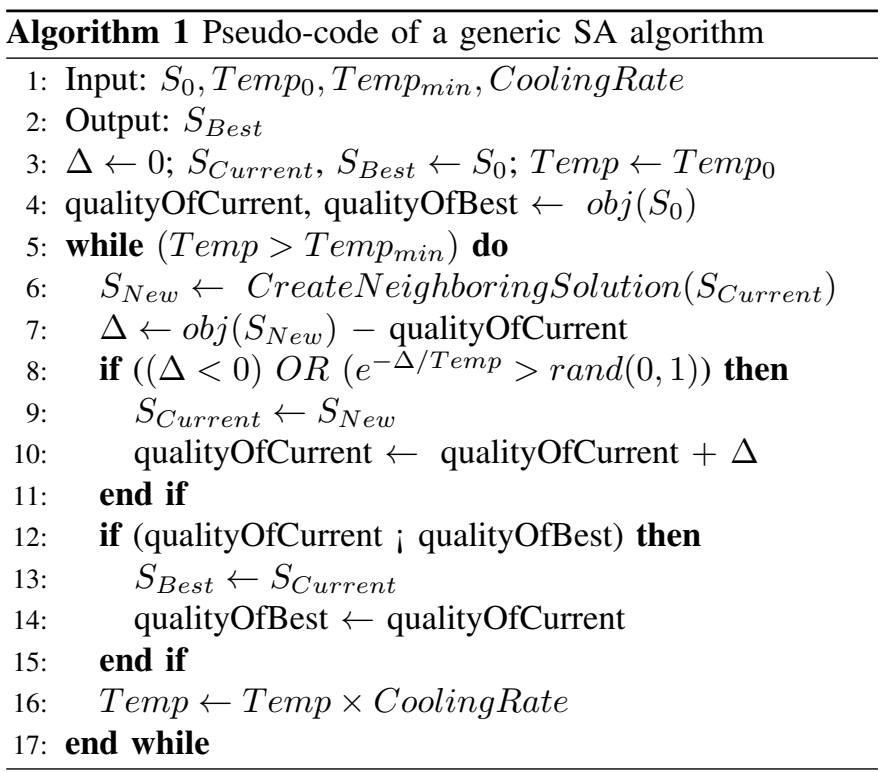

generated initial solution $\left(S_{0}\right)$. A new solution $\left(S_{N e w}\right)$ is generated using a predefined neighbourhood/move operator (CreateNeighboringSolution) within the vicinity of the solution in hand and compared to the original solution ( $S_{\text {Current }}$ ). Based on the solution's 'objective value', measuring its quality (fitness) $(o b j()$.$) and the current 'temperature'$ (Temp), new solution is either accepted or rejected (line 8 in Algorithm 1). An improving solution is always accepted. SA avoids becoming trapped at a local optimum by accepting worsening solutions with a probability based on temperature, change in the quality of the solution and current step using the Metropolis criterion [13] during the search process $(\operatorname{rand}()$ generates a uniform random number in between $[0,1])$. The temperature is initially set to a high value $\left(\operatorname{Temp}_{0}\right)$ that results in a high probability of inferior solutions being accepted. Then the temperature is slowly decreased (line 16 in Algorithm 1) over the course of a run, reducing the probability of inferior solutions being selected. This iterative process of creating new solutions, evaluating them and accepting/rejecting them is repeated until a termination criteria, i.e., maximum number of iterations or a final temperature $\left(T_{\min }\right)$ is reached. In our approach, we keep track of the best solution $\left(S_{B e s t}\right)$ visited by the SA approach and return that solution at termination.

\section{AN APPROACH FOR SOLVING A SUPPLy CHAIN MANAGEMENT PROBLEM}

In this study, we describe a two-stage fuzzy based optimisation approach to solve a supply chain management problem. The first stage of ranking suppliers using an IT2FS method is followed by invocation of a simulated annealing approach to optimize both cost and supplier risk, simultaneously.

\section{A. Stage One: Ranking of Suppliers}

This stage aims to provide an appropriate way to rank suppliers by identifying the criteria which have played an essential role in supplier selection, and evaluating supplier performance with respect to the selection criteria. 
TABLE I

LINGUISTIC WEIGHTS OF THE ATTRIBUTES REPRESENTED BY INTERVAL TYPE-2 FUZZY SETS

\begin{tabular}{ll}
\hline \hline Linguistic terms & Interval Type-2 fuzzy sets \\
\hline \hline Low importance & $((0.0,0.0,0.2,0.3),(0.0,0.0,0.2,0.5))$ \\
Moderate importance & $((0.3,0.4,0.4,0.5),(0.1,0.4,0.4,0.7))$ \\
High importance & $((0.5,0.6,0.6,0.7),(0.3,0.6,0.6,0.9))$ \\
Very High importance & $((0.7,0.8,1.0,1.0),(0.5,0.8,1.0,1.0))$ \\
\hline
\end{tabular}

TABLE II

LINGUISTIC PERFORMANCE RATES REPRESENTED INTERVAL TYPE-2 FUZZY SETS

\begin{tabular}{ll}
\hline \hline Linguistic terms & Interval Type-2 fuzzy sets \\
\hline \hline Poor & $((0,0,2,3),(0,0,2,5))$ \\
Good & $((3,4,4,5),(1,4,4,7))$ \\
Very Good & $((5,6,6,7),(3,6,6,9))$ \\
Excellent & $((7,8,10,10),(5,8,10,10))$ \\
\hline
\end{tabular}

1) Fuzzy Membership Functions: In work by Ordoobadi [19] decision makers considered two attributes important when evaluating suppliers, the weight of the selection criteria and the rating of each supplier. Turk et al. [25] extended this work, analysing uncertainty in the supplier selection problem using IT2FS.

To establish the importance of each criterion experts are asked to rate each with one of the following linguistic weights: 'low importance', 'moderate importance', 'high importance' and 'very high importance' [19].

Table I shows the parameters of the IT2 membership functions used to represent each of the linguistic weights. The numeric scale defined between 0 and 1 corresponded the fuzzy numbers.

Table I shows values of trapezoidal T2FS. A trapezoidal T1FS can be defined by four parameters as $a, b, c, d$ in which $a<b<c<d$. In Table I, IT2FS are described using values of the upper and lower membership functions. The second column gives the $a, b, c$ and $d$ values in sequence for each membership function for the lower and upper membership functions of the IT2FS.

In addition, the performance of a supplier with consideration for each criterion was elicited by asking experts to assign one of the following linguistic weights: 'excellent', 'very good', 'good' and 'poor'. The numeric scale defined between 0 and 10 corresponded to the fuzzy numbers of each criterion value [19]. The IT2FS are created in the same manner as explained previously for modelling the importance weights, and their values are illustrated in Table II.

2) Proposed Method for Ranking Suppliers: To evaluate suppliers and obtain their ranks the following steps are taken:

1) Trapezoidal IT2FS are generated using information about the importance of the criteria selected by the decision makers. Let $w_{i}$ denotes the fuzzy importance

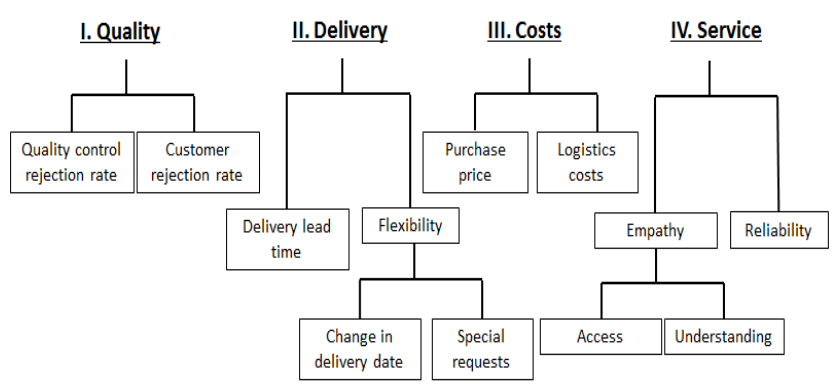

Fig. 1. The criteria and sub-criteria used for selection of suppliers [19].

weight of criterion $i$ where $i=1,2, \ldots, 10$. For instance, if a criterion's importance weight is 'low' then $w_{i}$ is denoted as $((0.0,0.0,0.2,0.3),(0.0,0.0,0.2,0.5))$ as indicated in Table I.

2) as shown in Figure 1, all nodes on the same branch are multiplied by previous node. As an example, $w_{1}$ is computed by multiplying the importance weight of the delivery by importance weight of delivery lead time as;

$$
\begin{gathered}
w_{1}=((0.5,0.6,0.6,0.7),(0.3,0.6,0.6,0.9)) \\
((0.5,0.6,0.6,0.7),(0.3,0.6,0.6,0.9)) \\
=((0.25,0.36,0.36,0.49) \\
(0.09,0.36,0.36,0.81))
\end{gathered}
$$

All weights are computed in the same manner.

3) The linguistic terms describing suppliers' performance are dealt with in the same way as criteria importance (Step 1).

4) The aggregate fuzzy set for each supplier is calculated by multiplying the fuzzy performance rates matrix by the fuzzy importance weights.

5) Centroid type-reduction and defuzzification methods are used to convert fuzzy values into crisp values.

\section{B. Stage Two: Inventory Planning with Consideration of Sup- plier Risk}

The problem used in this study captures the dynamics of the production of multiple products that are made up of different components. There are multiple suppliers, manufacturing plants and potential customers. The planning horizon for the inventory is discretized into time periods. The initial period relies on the initial stock levels to satisfy the demand, while the subsequent periods require inventory planning to meet the demand in a cost effective manner with a reduced supplier risk.

The following assumptions are made in this study:

- All suppliers can supply all plants with any of the components, each plant can supply any product to any customer.

- Each supplier/production plant has a fixed capacity for each component/product. 
TABLE III

NOTATION FOR DECISION VARIABLES
TABLE IV

NOTATION FOR PARAMETERS

\begin{tabular}{|c|c|c|c|}
\hline Variable & Meaning & Notation & Meaning \\
\hline$X_{(m, i, j, t)}$ & Amount of component $m$ from supplier $i$ to plant $j$ in period $t$ & $\alpha(m, i)$ & Capacity of supplier $i$ for component $m$ \\
\hline$Y_{(l, j, k, t)}$ & Amount of product $l$ from plant $j$ to customer $k$ in period $t$ & $\beta(m, i)$ & Cost of component $m$ at supplier $i$ \\
\hline$I c_{(m, j, t)}$ & Inventory of component $m$ at plant $j$ in period $t$ & $\beta_{T}(m, i, j)$ & Transportation cost of component $m$ from supplier $i$ to plant $j$ \\
\hline$I p_{(l, j, t)}$ & Inventory of product $l$ at plant $j$ in period $t$ & $\beta_{O}(m, i)$ & Order cost of component $m$ from supplier $i$ \\
\hline$I S c_{(m, j)}$ & Initial stock of component $m$ at plant $j$ & $D_{s}(i, j)$ & Distance from supplier $i$ to plant $j$ \\
\hline$I S p_{(l, j)}$ & Initial stock of product $l$ at plant $j$ & $R(i)$ & Rank of supplier $i$ \\
\hline \multirow{14}{*}{\multicolumn{2}{|c|}{$\begin{array}{l}\text { - The total cost of supply chain operation for the period } \\
\text { of inventory planning consists of batch (order) cost, } \\
\text { production cost, transportation cost, inventory holding } \\
\text { cost and stock out cost. } \\
\text { - Each product has a fixed production cost. } \\
\text { - Stock out cost is considered when an order is not in stock. } \\
\text { In the case of an inability to satisfy demand, items are } \\
\text { purchased at full retail price from a competing producer. } \\
\text { - The distances among suppliers, buyers and plants are } \\
\text { fixed and known. } \\
\text { - Supply chain dynamics is considered as a discrete-time } \\
\text { process. }\end{array}$}} & $\operatorname{Ri}(i)$ & Risk of supplier $i$ \\
\hline & & $\theta(l, j)$ & Capacity of plant $j$ for product $l$ \\
\hline & & $\gamma(l, j)$ & Cost of manufacturing product $l$ in plant $j$ \\
\hline & & $\gamma_{O}(l, j)$ & Setup cost of product $l$ in plant $j$ \\
\hline & & $\sigma(l, j)$ & Percentage of holding cost of product $l$ in plant $j$ \\
\hline & & $\gamma_{T}(l, j, k)$ & Transportation cost of product $l$ from plant $j$ to customer $k$ \\
\hline & & $\gamma_{p I}(l, j)$ & Inventory cost of product $l$ in plant $j$ \\
\hline & & $\gamma_{c I}(m, j)$ & Inventory cost of component $m$ in plant $j$ \\
\hline & & $\gamma_{p S}(l, j)$ & Shortage cost of product $l$ in plant $j$ \\
\hline & & $\gamma_{c S}(m, j)$ & Shortage cost of component $l$ in plant $j$ \\
\hline & & $D_{p}(j, k)$ & Distance from plant $j$ to customer $k$ \\
\hline & & $\omega(l, k, t)$ & Demand of customer $k$ for product $l$ for each period $t$ \\
\hline & & $\lambda(l, k)$ & Selling price of product $l$ for customer $k$ \\
\hline & & $m(l, k)$ & Missing amount of product $l$ for customer $k$ \\
\hline
\end{tabular}

- A periodic review policy is assumed.

- Initial stock level is determined in the beginning to satisfy orders in the initial period.

1) Problem Formulation: A supplier, manufacturing plant and customer is denoted by $i, j$ and $k$, respectively. Additionally, a product, denoted by $l$, is manufactured using $m$ components. Each discrete time period is indicated by $t$. Tables III and IV summarizes the inventory data and operational variables used in the model.

In this study, there are two objectives to optimize; (i) minimise exposure to risk due to the supplier selection and (ii) minimize the total cost of the supply chain, subject to a set of constraints. The weighted sum and Tchebycheff scalarisation are used to convert the bi-objective problem of minimizing the vector TotalFitness $(T F)$ into a scalar problem:

$$
\text { minimise } \quad \mathrm{TF}=w_{1} T R+w_{2} T C
$$

where $T R$ is the total risk associated with a supply chain (Equation 9), $T C$ is the total cost (Equation 9), their respective weights are $w_{1}$ and $w_{2}$ and $w_{1}+w_{2}=1$. On the other hand, Tchebycheff problem is of the form:

$$
\begin{aligned}
& \operatorname{minimise} \mathrm{TF}=\max \left\{w_{1}\left(T R-\mu_{T R}\right) /\left(\max _{T R}-\mu_{T C}\right),\right. \\
& \left.w_{2}\left(T C-\mu_{T C}\right) /\left(\max _{T C}-\mu_{T C}\right)\right\}
\end{aligned}
$$

where $\mu_{T R}$ is the utopian total risk associated with a supply chain, $\mu_{T C}$ is the e ideal total cost, $\max _{T} R, \max _{T} C$ are the maximum values in 100 experiments for $T R$ and $T C$, the $\mathrm{m}$

their respective weights are $w_{1}$ and $w_{2}$ and $w_{1}+w_{2}=1$ [14].

$$
\begin{aligned}
T C= & \sum_{l} \sum_{j} \gamma_{p I}(l, j) I p_{(l, j, t)}+\sum_{m} \sum_{j} \gamma_{c I}(m, j) I c_{(m, j, t)} \\
& +\sum_{l} \sum_{j} \sum_{k} Y_{(l, j, k, t)} D_{p}(j, k) \gamma_{T}(l, j, k) \\
& +\sum_{m} \sum_{i} \sum_{j} X_{(m, i, j, t)} D_{s}(i, j) \beta_{T}(m, i, j) \\
& +\sum_{m} \sum_{i} \sum_{j} \beta_{O}(m, i) X_{(m, i, j, t)} \\
& +\sum_{l} \sum_{j} \sum_{k} \gamma_{O}(l, j) Y_{(l, j, k, t)} \\
& +\sum_{l} \sum_{j} \sum_{k} \gamma(l, j) Y_{(l, j, k, t)} \\
& +\sum_{l} \sum_{j} \gamma_{p S}(l, j) I p_{(l, j, t)} \\
& +\sum_{m} \sum_{j} \gamma_{c S}(m, j) I c_{(m, j, t)} \\
& +\sum_{l} \sum_{k} m(l, k) \lambda(l, k)
\end{aligned}
$$

for $\forall \mathrm{t}$.

$$
T R=\sum_{m} \sum_{i} \sum_{j} X_{(m, i, j, t)} R i(i)
$$

The equation 8 represents the total cost of supply chain under consideration. The first row of the objective function is the sum of the inventory cost for the components and products respectively. In the second and third rows, total transportation 
cost is represented for both components and products. The next line demonstrates order cost of components. In the following two lines, setup cost and production cost are described. The seventh and eighth lines show how the total shortage cost for components and products is computed. The final line shows the penalty cost if the production amount does not satisfy the demand of customers.

A given problem can be optimized using a generic optimisation algorithm with respect to the the weighted sum of two objectives, subject to the following constraints which model the computations of risk from suppliers, supplier capacities, plant capacities, and inventory-control of both components and products.

$$
R i(m)=\sum_{i} R(i) / R(m)
$$

Equation 10 shows the computation of a supplier risk coefficient for each supplier by normalising the ranks of suppliers shown in Table $\mathrm{V}$.

$$
\begin{array}{cc}
\sum_{j} X_{(m, i, j, t)} \leq \alpha(m, i) & \text { for } \forall \mathrm{m}, \mathrm{i}, \mathrm{t} \\
\sum_{k} Y_{(l, j, k, t)} \leq \theta(l, j) & \text { for } \forall \mathrm{l}, \mathrm{j}, \mathrm{t}
\end{array}
$$

Equation 11 is the capacity of the supplier for each period and Equation 12 depicts plant capacity for each period.

$$
\sum_{j} Y_{(l, j, k, t)} \leq \omega(l, k, t) \quad \text { for } \forall 1, \mathrm{k}, \mathrm{t}
$$

Equation 13 stipulates that the production quantities are not less than the order quantities of customer.

$$
\begin{aligned}
& \sum_{i} X_{(m, i, j, t)}+I S c_{(m, j)}= \\
& \sum_{l} \sum_{k} Y_{(l, j, k, t)}+I c_{(l, j, t)} \quad \text { for } \forall 1, \mathrm{j}, \mathrm{t}
\end{aligned}
$$

Equation 14 represents the inventory-control constraints for each component.

$$
\begin{array}{ll}
\sum_{j} \sum_{k} Y_{(l, j, k, t)}+\sum_{j} I S p_{(l, j)}= & \\
\sum_{k} \omega(l, k, t)+\sum_{k} I p_{(k, l, t)} & \text { for } \forall 1, \mathrm{t}
\end{array}
$$

Equation 15 describes inventory-control constraints for each product.

\section{Simulated Annealing for Inventory Planning}

Simulated annealing approach requires design of several domain specific components, such as, solution representation, neighbourhood/move operator and algorithmic settings, such as, maximum number of iterations and cooling schedule. In this section, we provide the details of the SA approach used as a generic optimisation algorithm for solving the supply chain management problem described in the previous subsection. A real-valued solution representation is used in SA. A solution consists of a 4 dimensional array that represents an inventory plan. The dimensions correspond to the source node, destination node, component/product and time period respectively. Each element of the array contains a value in $[0,1]$ that denotes the amount of stock added to the inventory of a source node, for a destination node, of a product in a particular time period. For example, if currentPlan $[1,2,3,4]$ contains the value 0.5 , this indicates that source node 1 receives/produces $50 \%$ of its capacity of product 3 for destination node 2 in period 4 . This method of representing inventory ensures that a node can never supply/produce more than its capacity in one time period. Inventory values are also restricted by a minimum order quantity, and order quantities. For example, we might set a minimum order quantity of 100 for a particular product at a particular node, and then restrict orders to units of 100 (e.g. 500, 600, 700, 800 etc.).

TF (Equation 6) is used to evaluate the quality (fitness) of a candidate solution during the search process. Although it is a challenge to decide on how much weight to apply to each of the objectives [9]. These settings can be considered to reflect the attitude of a "decision maker" towards cost and risk in our study. More importantly and additionally, by running the algorithm with different weight settings, we can obtain a set of solutions indicating the trade-off between those objectives and the "decision maker" can choose one of the solutions as appropriate.

The algorithm generates an initial solution randomly, containing values in $[0,1]$ that represent the proportion of capacity added to each supply node for each destination node of each product in each time period. Then, SA attempts to improve this initial solution iteratively by applying a neighbourhood operator and then deciding whether to accept or reject the resultant solution. A parametrized neighbourhood operator is implemented for perturbing a solution. At each step, a new solution is generated by this operator modifying the settings of a number of randomly selected elements with random values and the number of elements being a discrete parameter value in between 1 and 10. This operator is an adaptive operator using reinforcement learning for parameter control [20]. The number of elements altered is chosen using a reinforcement learning method in which a set of counters is used to record the most successful number of changes. A utility value/score is maintained using a counter for each parameter setting. Initially, a random number of elements between 1 and 10 is chosen and the solution is altered accordingly. At any time, if the changes to the selected elements result in a non-worsening solution, the counter for that parameter value is incremented, otherwise it is decremented. When the next perturbation needs to be made, the algorithm looks for the number of elements with the maximum score, i.e., the most successful. If there are multiple numbers with the maximum value, one is chosen at random. This method encourages the SA algorithm to select the number of changes that has resulted in the largest ratio of improvements to changes detrimental to solutions. 
TABLE $\mathrm{V}$

SCORES FOUND FOR SUPPLIERS

\begin{tabular}{lccc}
\hline \hline Suppliers & Crisp Scores & Rank & Risk \\
\hline Supplier A & 10.29 & 2 & 3.24 \\
Supplier B & 23.08 & 1 & 1.45 \\
\hline
\end{tabular}

\section{NUMERICAL EXPERIMENTS}

\section{A. Experimental Setup}

The supply chain problem instance used in this study contains two different products that are made up of four different components. There are two suppliers (denoted as A and $\mathrm{B}$, two manufacturing plants and two potential customers. The planning horizon contains three discrete time periods.

In the first stage, the importance of each criterion and performance of suppliers with respect to each one are determined by the decision makers. Then these values are used in an Interval Type-2 fuzzy model to rank the suppliers as described in Section III-A.

We tested SA on the problem instance using the information from the previous stage considering 6 different weight settings for $w_{1}$, while $w_{2}=1.0-w_{1}$ yielding 6 different scenarios for optimizing $T F$ with weighted sum and Tchebycheff scalarisations of cost and risk objectives as provided in Equations 6 and 7, respectively. Each scenario Scn-1, Scn-2, Scn-3, Scn-4 and $\operatorname{Scn}-5$ uses $0.0,0.2,0.5,0.8$ and 1.0 as a setting for $w_{1}$, respectively. The last scenario (Scn-6) generates 100 random settings for $w_{1}$ in $(0,1]$ with $w_{2}=1.0-w_{1}$. A single SA run is performed with each setting generating 100 solutions. This experiment is performed to observe the trade-off solutions for the given instance. The cooling schedule for the temperature within SA is geometric, resulting in a slow and consistent reduction of temperature over the course of a run. The initial temperature is fixed as 10,000 . The cooling rate is computed to allow 100,000 iterations and then SA terminates. Each SA experiment is repeated 30 times, except for Scn-6.

\section{B. Results}

The stage one fuzzy approach described in Section III-A yields the output as shown in Table V. Supplier B has a better ranking than $\mathrm{A}$ due to low risk. This information is fed into the SA solver described in Section III-B for inventory planning.

SA indeed discovers high quality inventory plans based on the described model for those scenarios as shown in Table VI. Clearly, SA produces lower mean $T R$ values (higher $T C$ values) as the weight of total supplier risk increases (total cost of the supply chain operation decreases) when weighted sum is used. SA provides flexibility for the decision makers enabling them to try out different risk weightings (representing riskavoiding or risk-taking strategies), and observe how this choice influences inventory planning in terms of the total supplier risk and cost of the supply chain operation. It seems that the SA using Tchebycheff scalarisation spends more effort on the improvement of $T R$ as compared to $T C$.

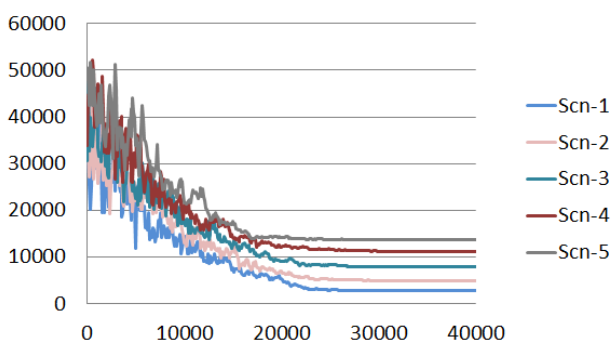

Fig. 2. Behaviour of the SA algorithm in a single run for each scenario using weighted sum scalarisation.
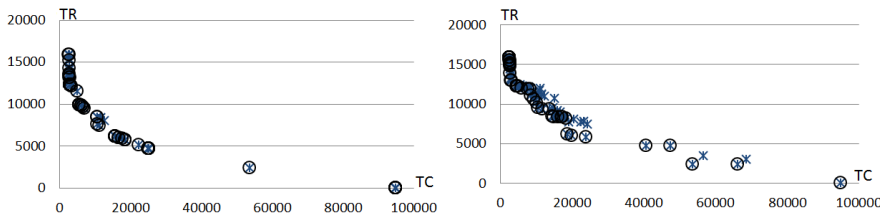

Fig. 3. $T R$ vs $T C$ from 100 runs (each with a random weight setting) of the SA algorithm based on weighted sum (on the left) and Tchebycheff (on the right) scalarisations. Circles indicate the pareto front.

As for the behaviour of the SA during the search process, Figure 2 provides the progress plot of the weighted sum based objective value $(T F)$ of the current solution in a single run for each scenario as an example. The objective value oscillates radically during the initial phases of the search process. This indicates that large moves are accepted initially as expected, and this behaviour settles down in time and the algorithm seems to reach a plateau for all scenarios. This is likely due to the chosen cooling rate and initial temperature. Although we tested a set of different values for parameter tuning purposes, we did not observe much performance improvement over what has been reported in here.

Figure 3 provides the scatter plot of $T R$ versus $T C$, obtained after applying the SA using weighted sum and Tchebycheff scalarisation approaches under Scn-6. The former approach produces a slightly better pareto front than the latter one with a hypervolume [28] of 0.75 versus 0.74 . In the overall, the results indicate that there is certainly a trade-off between risk and cost. The trade-off solutions can be obtained using a simple weighted sum approach via a generic SA optimisation algorithm. Hence, it is possible for decision makers to choose an appropriate strategy based on their aversion to risk and cost. The proposed system could be used in What-If scenarios to see how varying attitudes to risk affect inventory planning. A decision maker can alternatively choose a solution from the set of trade-off solutions.

\section{CONCLusions}

In this study, we provided a formulation for a supply chain management problem integrating supplier selection and inventory planning and proposed a two-stage solution method based on an Interval Type-2 fuzzy system and simulated annealing. 
TABLE VI

BEST AND AVERAGE RESULTS IN TERMS OF $T R, T C$ OBTAINED FROM 30 RUNS FOR EACH SCENARIO FOR WEIGHTED SUM AND TCHEBYCHEFF SCALARISATION

\begin{tabular}{cllll|lllll}
\hline & \multicolumn{4}{c}{ Weighted sum } & \multicolumn{4}{c}{ Tchebycheff } \\
\hline & \multicolumn{3}{c}{ Average } & \multicolumn{2}{c}{ Best } & \multicolumn{2}{c}{ Average } & \multicolumn{2}{c}{ Best } \\
\hline Scenario & $T R$ & $T C$ & $T R$ & $T C$ & $T R$ & TC & $T R$ & $T C$ \\
\hline Scn-1 & 14867 & 2769 & 15504 & 2630 & 14867 & 2769 & 15504 & 2630 \\
Scn-2 & 13004 & 2995 & 14605 & 2743 & 14557 & 3144 & 13706 & 3063 \\
Scn-3 & 12259 & 3151 & 12628 & 2908 & 14881 & 2756 & 15863 & 2640 \\
Scn-4 & 12179 & 3350 & 12628 & 2888 & 14875 & 2752 & 15853 & 2630 \\
Scn-5 & 11839 & 8145 & 12269 & 5600 & 11839 & 8145 & 12269 & 5600 \\
\hline
\end{tabular}

In the first stage, IT2FSs are used to rank suppliers for supplier selection. In the second stage, SA is used to minimize supplier risk and operational costs for inventory planning, simultaneously. SA performed well using a learning move operator with an adaptive parameter control. The proposed approach is capable of capturing the trade-off between risk and cost via scalarisation of both objectives, giving flexibility to the decision makers to choose from a set of trade-off solutions for supply chain management. We tested weighted sum and Tchebycheff scalarisation approaches and as a future work, we will investigate the performance of the other multi-objective optimisation approaches to tackle this problem.

\section{REFERENCES}

[1] Introduction to multiple attribute decision-making (madm) methods. In Decision Making in the Manufacturing Environment, Springer Series in Advanced Manufacturing, pages 27-41. Springer London, 2007.

[2] Shyi-Ming Chen and Li-Wei Lee. Fuzzy multiple attributes group decision-making based on the interval type-2 topsis method. Expert Syst. Appl., 37(4):2790-2798, April 2010.

[3] Shyi-Ming Chen and Li-Wei Lee. Fuzzy multiple attributes group decision-making based on the ranking values and the arithmetic operations of interval type-2 fuzzy sets. Expert Syst. Appl., 37(1):824-833, January 2010.

[4] S.H Ghodsypour and C OBrien. The total cost of logistics in supplier selection, under conditions of multiple sourcing, multiple criteria and capacity constraint. International Journal of Production Economics, 73(1):15 - 27, 2001. Supply Chain Management.

[5] Yanbing Gong. Fuzzy multi-attribute group decision making method based on interval type-2 fuzzy sets and applications to global supplier selection. International Journal of Fuzzy Systems, 15(4), 2013.

[6] Sarah Greenfield, Francisco Chiclana, Robert John, and Simon Coupland. The sampling method of defuzzification for type-2 fuzzy sets: Experimental evaluation. Information Sciences, 189(0):77 - 92, 2012.

[7] William Ho, Xiaowei Xu, and Prasanta K. Dey. Multi-criteria decision making approaches for supplier evaluation and selection: A literature review. Eur. J. Oper. Res., 202(1):16 - 24, 2010.

[8] M. Kalender, A. Kheiri, E. Özcan, and E. K. Burke. A greedy gradient-simulated annealing selection hyper-heuristic. Soft Computing, 17(12):2279-2292, 2013.

[9] I.Y. Kim and O.L. de Weck. Adaptive weighted sum method for multiobjective optimization: a new method for pareto front generation. Structural and Multidisciplinary Optimization, 31(2):105-116, 2006.

[10] S. Kirkpatrick, C. D. Gelatt, and M. P. Vecchi. Optimization by simulated annealing. SCIENCE, 220(4598):671-680, 1983.

[11] Jerry M Mendel and RI Bob John. Type-2 fuzzy sets made simple. Fuzzy Systems, IEEE Transactions on, 10(2):117-127, 2002.

[12] Jerry M. Mendel, Robert John, and Feilong Liu. Interval type-2 fuzzy logic systems made simple. IEEE T. Fuzzy Systems, 14(6):808-821, 2006.
[13] Nicholas Metropolis, Arianna W. Rosenbluth, Marshall N. Rosenbluth, Augusta H. Teller, and Edward Teller. Equation of state calculations by fast computing machines. Journal of Chemical Physics, 21:1087-1092, 1953.

[14] Kaisa Miettinen. Nonlinear Multiobjective Optimization. Kluwer Academic Publishers, Boston, MA, 1999.

[15] Simon Miller, Mario Gongora, Jon Garibaldi, and Robert John. Interval type-2 fuzzy modelling and stochastic search for real-world inventory management. Soft Computing, 16(8):1447-1459, 2012.

[16] Simon Miller, Mario Gongora, and Robert John. Interval type-2 fuzzy modelling and simulated annealing for real-world inventory management. In Hybrid Artificial Intelligence Systems 2011 (HAIS2011) 23-25 May, 2011 Wroclaw, Poland, pages 231-238, 2011.

[17] Simon Miller and Robert John. An interval type-2 fuzzy multiple echelon supply chain model. Knowledge-Based Systems, 23(4):363 368, 2010. Artificial Intelligence 2009 AI-2009 The 29th $\{$ SGAI $\}$ International Conference on Artificial Intelligence.

[18] D. Mohammaditabar, S. H Ghodsypour, and C O'Brien. A supplier selection model with classification and joint replenishment of inventory items.

[19] Sharon M. Ordoobadi. Development of a supplier selection model using fuzzy logic. International Journal of Supplier Chain Management, 14(4):314 - 327, 2009

[20] Ender Özcan, Mustafa Misir, Gabriela Ochoa, and Edmund K. Burke. A reinforcement learning - great-deluge hyper-heuristic for examination timetabling. Int. J. of Applied Metaheuristic Computing, 1(1):39-59, 2010.

[21] M. Parhizkari, M. Amiri, and M. Mousakhani. A multiple criteria decision making technique for supplier selection and inventory management strategy: A case of multi-product and multi-supplier problem. Decision Science Letters, 2(3):185 - 190, 2013.

[22] Jafar Rezaei and Mansoor Davoodi. A deterministic, multi-item inventory model with supplier selection and imperfect quality. Applied Mathematical Modelling, 32(10):2106 - 2116, 2008.

[23] B. Suman and P. Kumar. A survey of simulated annealing as a tool for single and multiobjective optimization. Journal of the Operational Research Society, 57(18):1143-1160, 2006.

[24] Douglas J. Thomas and Paul M. Griffin. Coordinated supply chain management. Eur. J. Oper. Res., 94(1):1 - 15, 1996.

[25] S. Türk, R. John, and E. Özcan. Interval type-2 fuzzy sets in supplier selection. In Computational Intelligence (UKCI), 2014 14th UK Workshop on, pages 1-7, Sept 2014.

[26] Zhibin $\mathrm{Wu}$ and Yihua Chen. The maximizing deviation method for group multiple attribute decision making under linguistic environment. Fuzzy Sets Syst., 158(14):1608-1617, July 2007.

[27] L. Zadeh. The consept of a linguistic variable and its applications to approximate reasoning. Inform Science, 8:199 - 249, 1975.

[28] E. Zitzler and L. Thiele. Multiobjective evolutionary algorithms: a comparative case study and the strength pareto approach. Evolutionary Computation, IEEE Transactions on, 3(4):257-271, Nov 1999. 